\title{
PRACTICAL CALCULATION OF FLEXIBLE MEMBERS WITH THE USE OF NON-LINEAR DEFORMATION MODEL AS EXEMPLIFIED BY TYPICAL GIRDER RGD 4.56-90
}

\author{
Eres Opbul ${ }^{1}$, Dmitrii Dmitriev², Phan Van Phuc ${ }^{1,3}$ \\ ${ }^{1}$ Saint Petersburg State University of Architecture and Civil Engineering \\ 2-ya Krasnoarmeiskaya st., 4, St. Petersburg, Russia \\ 2 "TEKTON-SPB" LLC \\ Oktyabrskaya nab., 104, St. Petersburg, Russia \\ ${ }^{3}$ Vinh University \\ 182 Le Duan, Ben Thuy, Vinh City, Vietnam \\ ${ }^{1}$ phucprodhv@gmail.com
}

\begin{abstract}
The article is devoted to practical calculation of strength of flexible members made on the basis of non-linear deformation model taking into account experimental diagrams of materials deformation. The relative deformations are determined in the proposed calculation depending on maximum flexion of a member. The calculation of maximum flexion of a member will be performed using two iteration methods. The basic calculation formula, original block diagrams of methods and comparison of calculation parameters proceeding from results of practical calculations made as exemplified by typical girder are provided.
\end{abstract}

\section{Keywords}

Deformation model, non-linear calculation, deformation diagram, iteration, stiffness matrix, stress diagram, neutral line, reinforcement.

\section{Introduction}

Obviously, the calculation of construction structures with the use of non-linear deformation model (NLDM) qualifies as check-and-control or testing method. In this case the calculation according to NLDM will be performed with definite force (external or internal) values, reinforcement (of concrete) and member geometrical dimensions (Boujelben, Ibrahimbegovic, 2017; Jagtap et al., 2018).

This article presents two methods of iteration calculations. The purpose of iteration process consists in determining maximum flexion of a member and location of relative deformations in target points of sections depending on the target flexure (Patni, 2018; Mao, Zhang, 2018).

The target flexure is determined in the first method with finding a new position of neutral line, in the second method it depends on the matrix of stiffening behavior of every small area.

The first iteration method of calculation with respect to steel fiber reinforced-concrete structures has been described in the works (Morozov, Opbul, 2016; Opbul et al., 2017). Described below is the second method of nonlinear calculation with due account of paragraphs 8.1.208.1.29, 3.72-3.75 given in standards (TsRIIPromzdaniy OJSC, 2005; Minstroy RF, 2013) (In order to contract the 
volume of article, the first three and the last (eleventh) iterations of calculations only are given).

\section{Diagrams of materials deformation}

A typical girder RGD 4.56-90: concrete B40, reinforcement $A 400$ are being considered. Ref. Fig. 3 for girder geometrical dimensions and reinforcement.

The calculation formulae have been acquired according to (TsRIIPromzdaniy OJSC, 2005; Minstroy RF, 2013) and shown in Fig. 1, 2 to determine stresses and stress-strain moduli.

Judgments and assumptions: plane sections hypothesis is true; for determining center of gravity of the given girder cross-section and for the first method of iteration calculation the girder material works in the elastic stage (there are no cracks, the moduli of small areas equal the initial modulus of elasticity).

a) Calculation formulae of stresses and moduli for concrete of class $B 40$

In case of concrete compression:

- $0<\varepsilon_{b i}<150 \cdot 10^{-5}: \sigma_{b i}=R_{b} \cdot \frac{\varepsilon_{b i}}{150 \cdot 10^{-5}} \mathrm{MPa} ;$

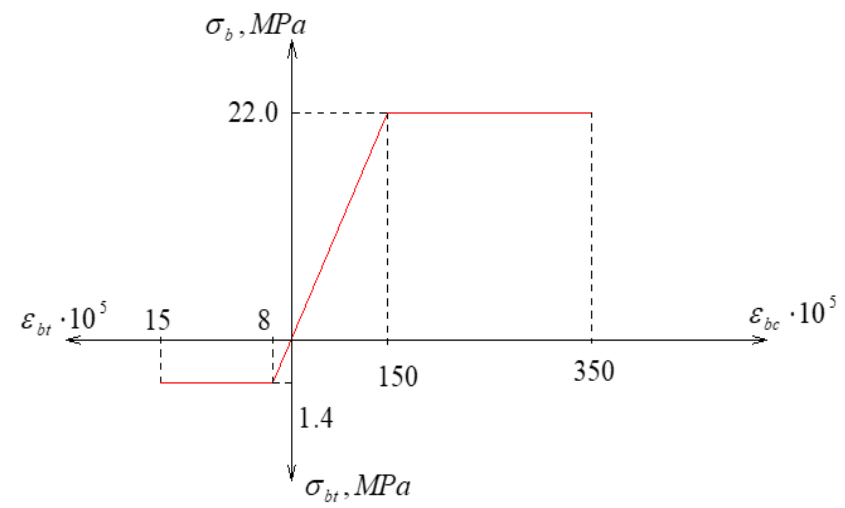

Figure 1. Diagram of Concrete Condition

$E_{b i}^{\prime}=$ const $=\frac{R_{b}}{150 \cdot 10^{-5}}=14666.7 \mathrm{MPa}$

- $150 \cdot 10^{-5}<\varepsilon_{b i}<350 \cdot 10^{-5}: \sigma_{b i}=R_{b}=22 \mathrm{MPa}$;

$E_{b i}^{\prime}=\frac{R_{b}}{\varepsilon_{b i}}=\frac{22}{\varepsilon_{b i}} \mathrm{MPa}$

In case of concrete expansion:

- $\quad 0<\varepsilon_{b t i}<8 \cdot 10^{-5}: \sigma_{b t i}=R_{b t} \cdot \frac{\varepsilon_{b t i}}{8 \cdot 10^{-5}} ;$

$E_{b t i}^{\prime}=\frac{R_{b t}}{8 \cdot 10^{-5}}=\frac{1.4}{8 \cdot 10^{-5}}=17500 \mathrm{MPA}$

- $8 \cdot 10^{-5}<\varepsilon_{b t i}<15 \cdot 10^{-5}: \sigma_{b t i}=R_{b t}=1.4 \mathrm{MPa}$;

$E_{b t i}^{\prime}=\frac{1.4}{\varepsilon_{b t i}} \mathrm{MPa}$

b) Calculation formula of stresses and moduli for reinforcement in case of expansion and contraction:

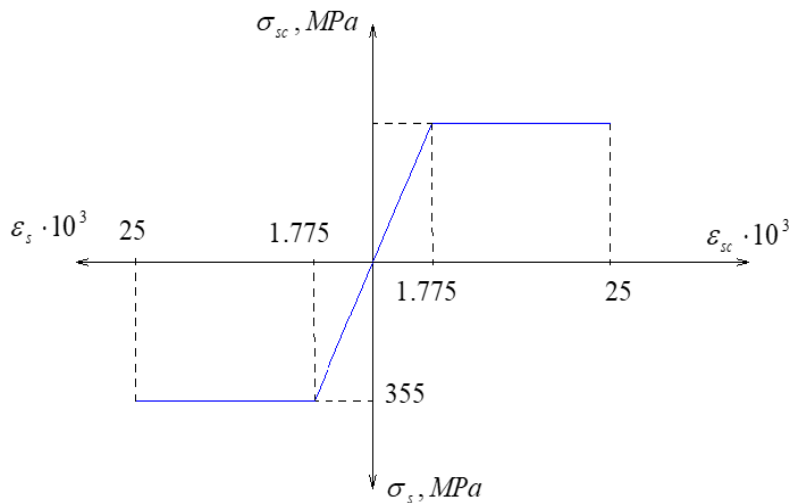

Figure 2. Diagram of Reinforcement Condition

- $\quad 0<\varepsilon_{s i}<1.775 \cdot 10^{-3}: \sigma_{s i}=R_{s} \cdot \frac{\varepsilon_{s i}}{\varepsilon_{s 1}}=355 \cdot \frac{\varepsilon_{s i}}{1.775 \cdot 10^{-3}} \mathrm{MPa}$, where $\varepsilon_{s 1}=\frac{R_{s}}{E_{s}}=\frac{355}{2 \cdot 10^{5}}=1.775 \cdot 10^{-3} \quad E_{s}^{\prime}=E_{s}$

- $1.775 \cdot 10^{-3}<\varepsilon_{s i}<25 \cdot 10^{-3}: \sigma_{s i}=R_{s}=355 \mathrm{MPa}$,

$E_{s}^{\prime}=\frac{R_{s}}{\varepsilon_{s i}}=\frac{355}{\varepsilon_{s i}}, \mathrm{MPa}$

\section{Basic calculation formulae and block diagram of} the first method

A) Center of gravity of a given cross section:

$y_{0}=\frac{S_{r e d}}{A_{\text {red }}}$

B) Reduced static moment:

$S_{r e d}=\sum A_{b i} y_{b i}+\sum \alpha \cdot A_{s i} y_{s i}$,

where $A_{b i^{\prime}} A_{s i}$ - area of section of $i$-n small area (layer) of concrete and $i$-n reinforcement, accordingly; $y_{b i} y_{s i}$ distances from extreme stretched fiber, accordingly, to the center of gravity of $i-n$ small area of concrete and $i$-n reinforcement, $\alpha$ - modular ratio of reinforcement into concrete.

C) Reduced cross-section area:

$A_{\text {red }}=\sum b_{b i} \cdot \Delta_{b i}+\sum \alpha \cdot A_{s i}$,

where $b_{b i} \Delta_{b i}$ - width and height (thickness) of $i$-n concrete small area, accordingly.

D) New position of neutral line (NL):

$y_{0 j}=\frac{\sum E_{b i}^{\prime} A_{b i} y_{b i}+\sum E_{s i}^{\prime} A_{s i} y_{s i}}{\sum E_{b i}^{\prime} A_{b i}+\sum E_{s i}^{\prime} A_{s i}}$,

where, $E_{b i}^{\prime}, E_{s i}^{\prime}$ - moduli of concrete and reinforcement deformation.

E) Member flexure:

$\frac{1}{r_{j}}=\frac{M}{E I_{\text {red }}}=\frac{M}{\sum E_{b i}^{\prime} A_{b i}\left(y_{b i}^{\prime}\right)^{2}+\sum E_{s i}^{\prime} A_{s i}\left(y_{s i}^{\prime}\right)^{2}}$ 


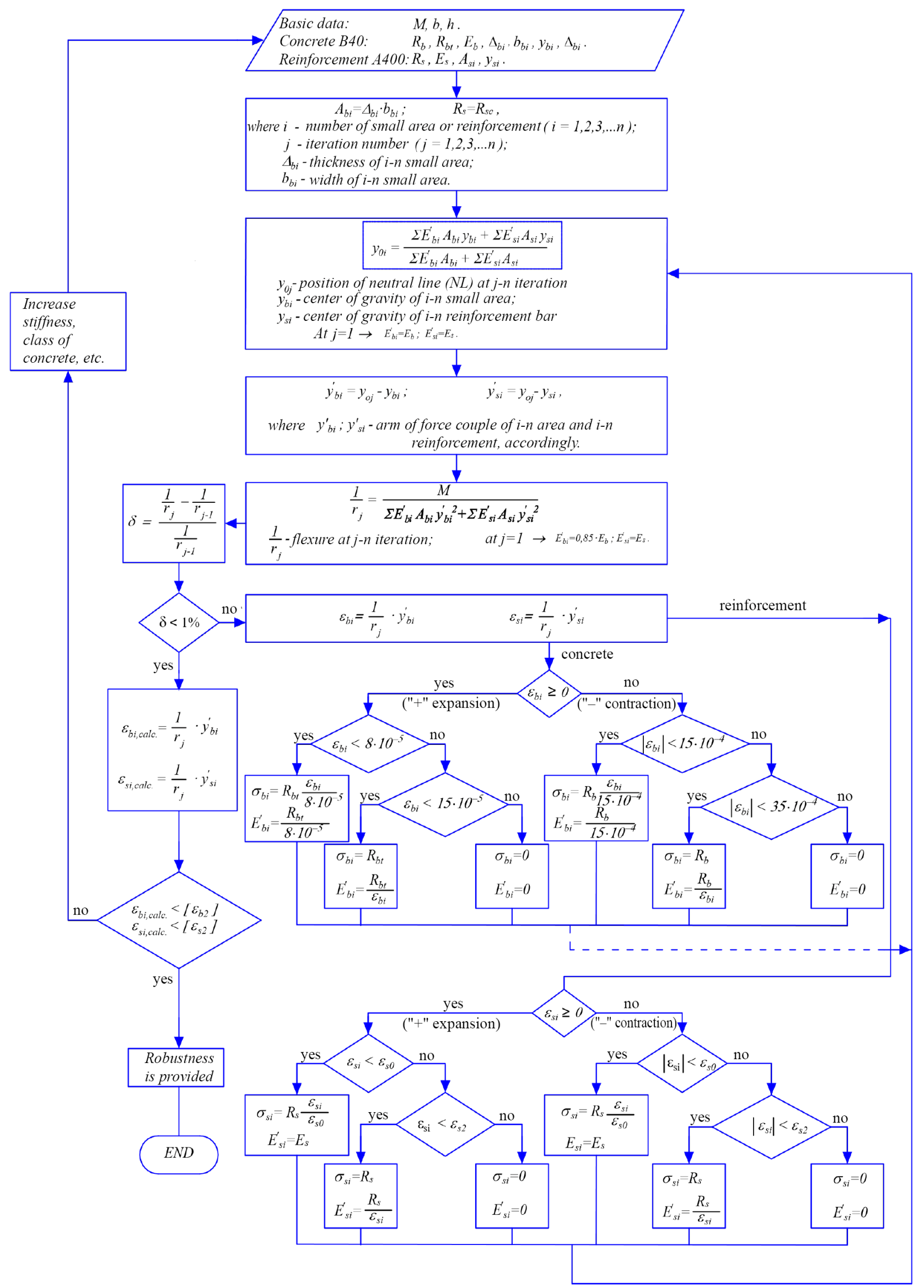

Figure 3. Block Diagram of the First Method 


\section{Architecture and Engineering Volume 3 Issue 3}

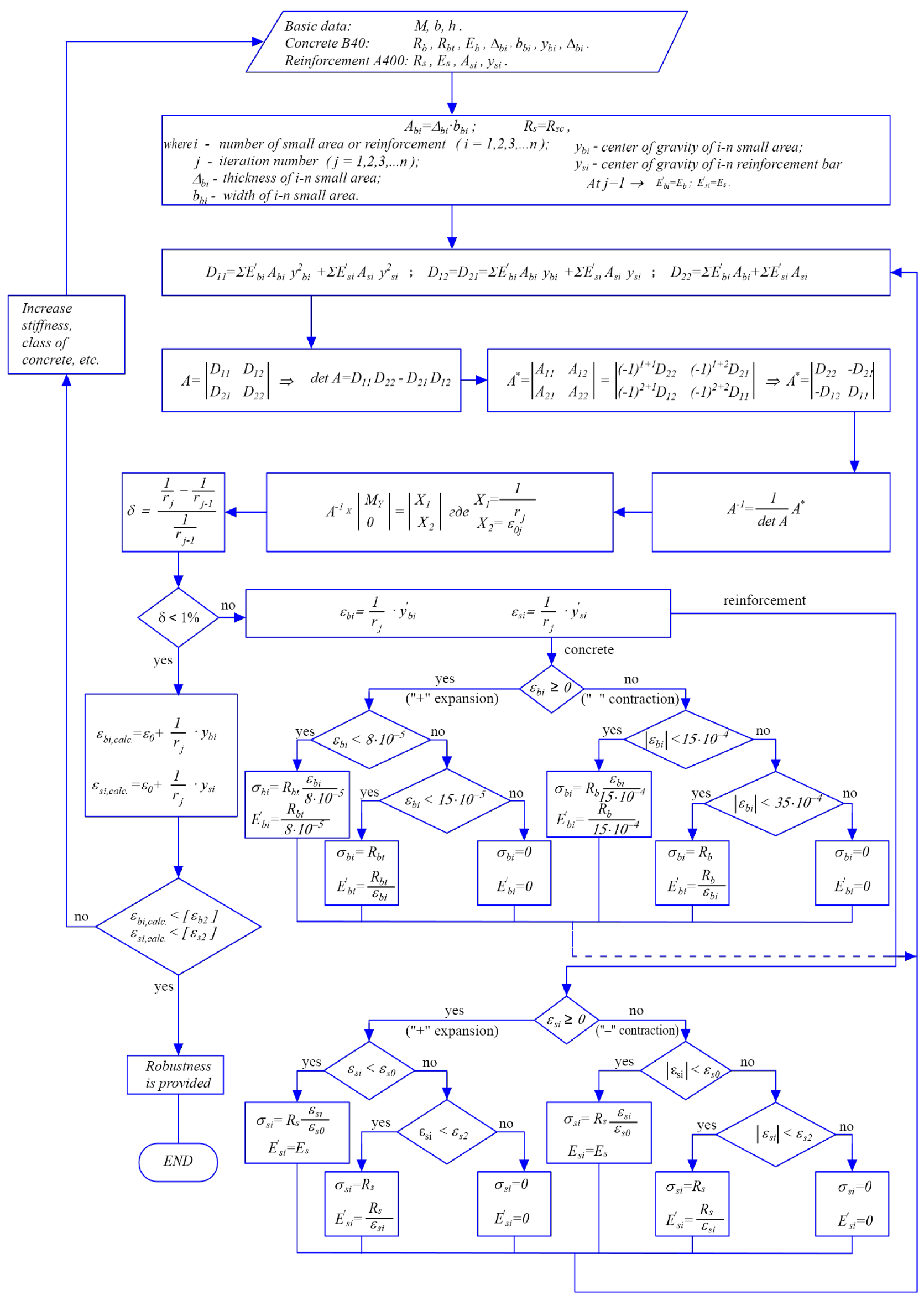

Figure 4. Block Diagram of the Second Iteration Method 
where, $y_{b i}^{\prime}=y_{0 j}-y_{b i}, y_{s i}^{\prime}=y_{0 j}-y_{s i}-$ arm of force couple for small areas of concrete and reinforcement, accordingly.

F) Condition when the maximum design flexure occurs $\frac{1}{r_{j, \text { calc }}}$ :

$\delta=\frac{\frac{1}{r_{j}}-\frac{1}{r_{j-1}}}{\frac{1}{r_{j-1}}} \cdot 100 \% \leq 1 \%$

\section{G) Deformations}

$\varepsilon_{b i}=\frac{1}{r_{j}} \cdot y_{b i}^{\prime} ; \varepsilon_{s i}=\frac{1}{r_{j}} \cdot y_{s i}^{\prime}$

H) Condition of strength, when compliance is necessary:

- $\quad$ with respect to deformations:

$\varepsilon_{b i, c a l c}<\left[\varepsilon_{b 2}\right] ; \varepsilon_{\text {si, calc }}<\left[\varepsilon_{s 2}\right]$,

where $\quad \varepsilon_{b i, \text { calc }}=\frac{1}{r_{j, \text { calc }}} \cdot y_{b i}^{\prime}, \quad \varepsilon_{s i, c a l c}=\frac{1}{r_{j, \text { calc }}} \cdot y_{s i}^{\prime} \quad-$ design deformations; $\left[\varepsilon_{b 2}\right], \quad\left[\varepsilon_{s 2}\right]$-permissible deformations according to [1].

- $\quad$ with respect to forces according to formula 3.144:

$M_{\text {calc }}=\sum \sigma_{b i} A_{b i} y_{b i}^{\prime}+\sum \sigma_{s i} A_{s i} y_{s i}^{\prime} \geq M_{u l t}$

Figure 3 shows an original block diagram of the first calculation method acquired in the way of iteration.

Calculation Formulae and Block Diagram of the Second Method

A) We use calculation equation $8.26 \div 8.52$ (Morozov, Opbul, 2016), where three stiffness characteristics only get defined in case of pure flexure:

$\left\{\begin{array}{c}D_{11}=\sum_{i} E_{b i}^{\prime} A_{b i}\left(y_{b i}^{\prime}\right)^{2}+\sum_{i} E_{s i}^{\prime} A_{s i}\left(y_{s i}^{\prime \prime}\right)^{2} \\ D_{13}=D_{31}=\sum_{i} E_{b i}^{\prime} A_{b i} y_{b i}^{\prime}+\sum_{i} E_{s i}^{\prime} A_{s i} y_{s i}^{\prime} \\ D_{33}=\sum_{i} E_{b i}^{\prime} A_{b i}+\sum_{i} E_{s i}^{\prime} A_{s i}\end{array}\right.$

B) Further, matrix determinant $(\operatorname{det} A)$ of $A=\left|\begin{array}{cc}D_{11} & D_{13} \\ D_{31} & D_{33}\end{array}\right|$ type will be defined, wherefrom:

$\operatorname{det} A=D_{11} \cdot D_{33}-D_{31} \cdot D_{13}$
Inverse matrix $A^{-1}=\frac{1}{\operatorname{det} A} \times A^{*}$

where $A^{*}=\left|\begin{array}{ll}A_{11} & A_{12} \\ A_{21} & A_{22}\end{array}\right|$ matrix of cofactors equal to:

$A_{11}=(-1)^{1+1} \times D_{33}=D_{33}$

$A_{12}=(-1)^{1+2} \times D_{31}=-D_{31}$

$A_{21}=(-1)^{2+1} \times D_{13}=-D_{13}$

$A_{22}=(-1)^{2+2} \times D_{11}=D_{11}$

C) Product of matrices gets calculated in the form of:

$A^{-1} \times\left|\begin{array}{c}M \\ 0\end{array}\right|=\left|\begin{array}{l}x_{1} \\ x_{2}\end{array}\right|$,

where $x_{1}=\frac{1}{\rho_{j}} ; \quad x_{2}=\varepsilon_{0}$

D) Relative deformations in concrete and reinforcement get determined according to formulae 8.29, 8.30 [1] taking into account formulae (16):

$\varepsilon_{b i}=\varepsilon_{0}+\frac{1}{\rho_{j}} \cdot y_{b i}^{\prime}$

$\varepsilon_{s i}=\varepsilon_{0}+\frac{1}{\rho_{j}} \cdot y_{s i}^{\prime}$

E) Strength condition:

- $\quad$ with respect to deformations - ref. formula (8).

- $\quad$ with respect to forces according to formula 3.144 [2]:

$M_{\text {calc }}=\sum \sigma_{b i} A_{b i} y_{b i}+\sum \sigma_{s i} A_{s i} y_{s i} \geq M_{u l t}$

Figure 4 shows an original block diagram of the second iteration method.

\section{Determining girder load-carrying ability}

Basic data:

Class of concrete B40:

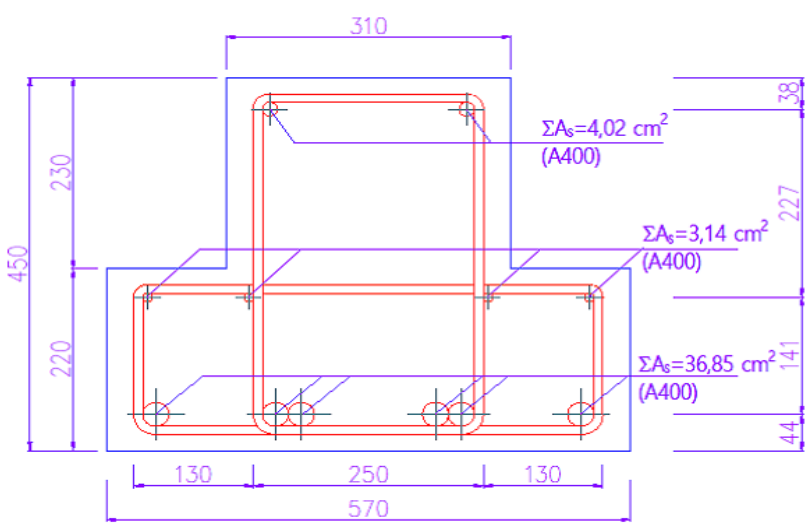

Figure 5. Girder Transversal Section 


\section{Architecture and Engineering Volume 3 Issue 3}

$$
R_{b}=22 \mathrm{MPa} ; R_{b t}=1.4 \mathrm{MPa} ; \quad E_{b}=36000 \mathrm{MPa}
$$

Longitudinal reinforcement of class A-III:

$$
R_{s}=355 \mathrm{MPa} ; R_{s c}=355 \mathrm{MPa} ; \quad E_{s}=2 \cdot 10^{5} \mathrm{MPa}
$$

Transversal section of $570 \times 450 \mathrm{~mm}$, girder length of 5,400 mm (Fig. 5).

Height of compressed zone:

$x=\frac{R_{s}\left(A_{s 1}+A_{s 2}-A_{s 3}\right)}{R_{b} b}=\frac{355 \cdot(3685+314-402)}{22 \cdot 310}=187 \mathrm{~mm}$

Position of the center of gravity of compressed zone:

$y_{x}=\frac{b \cdot \frac{x^{2}}{2}+\alpha \cdot A_{s 3} \cdot a_{s}^{\prime}}{b \cdot x+\alpha \cdot A_{s}}=\frac{310 \cdot \frac{187^{2}}{2}+5.26 \cdot 402 \cdot 38}{310 \cdot 187+5.26 \cdot 402}=92 \mathrm{~mm}$

Design load-carrying ability:

$M_{u l t}=R_{s}\left[A_{s 1}\left(h_{0}-y_{x}\right)+A_{s 2}\left(h_{0}-y_{x}-141\right)\right]=$

$=355 \cdot[3685 \cdot(406-92)+314 \cdot(406-92-141)]=430 \cdot 10^{6} \mathrm{~N} \mathrm{~m}$

Breakdown of transversal section to small areas and finding the center of gravity of every area

In order to calculate the transversal section of a member, we break it down randomly into small areas
(Fig. 6). We have thirteen small areas for our example. The numbering of areas begins from the lower stretched cross-section zone.

Table 1 gives: $i$ - order number of small area; $\Delta_{b i}, y_{b i}, b_{b i}, A_{b i}$ - height, distance from extreme fiber of the lower stretched zone to the center of gravity, width and area of $i$-n small area of concrete, accordingly; $E_{b i}^{\prime}=0.85 E_{b} \quad$ - modulus of concrete deformation; $y_{s i}, A_{s i}, E_{s i}^{\prime}=E_{s}$ - accordingly, distance from extreme fiber

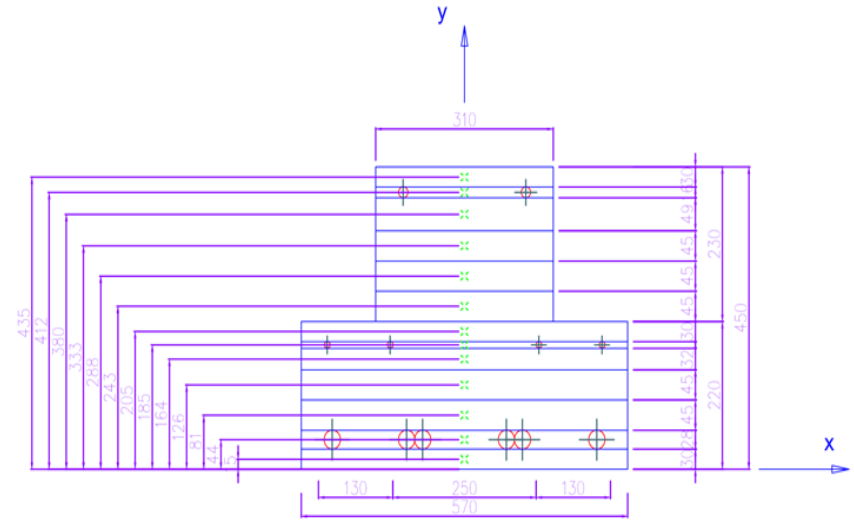

Figure 6. Breakdown of Transversal Section

Example of calculation according to the first iteration method

Table 1. Design parameters of small areas

\begin{tabular}{|c|c|c|c|c|c|c|c|c|c|}
\hline \multirow{2}{*}{$i$} & $\Delta_{b i}$ & $y_{b i}$ & $b_{b i}$ & $A_{b i}$ & $E_{b i}^{\prime}$ & $y_{s i}$ & $A_{s i}$ & $E_{s i}^{\prime}$ & $y_{o j}$ \\
\hline & $\mathrm{cm}$ & $\mathrm{cm}$ & $\mathrm{cm}$ & $\mathrm{cm}^{2}$ & $\mathrm{MPa}$ & $\mathrm{cm}$ & $\mathrm{cm}^{2}$ & $\mathrm{MPa}$ & $\mathrm{cm}$ \\
\hline 1 & 3 & 1,5 & 57 & 171 & $3,06 \mathrm{E}+04$ & & & & \\
\hline 2 & 2,8 & 4,4 & 57 & 122,8 & $3,06 \mathrm{E}+04$ & 4,4 & 36,8 & $2, \mathrm{E}+05$ \\
\hline 3 & 4,5 & 8,05 & 57 & 256,5 & $3,06 \mathrm{E}+04$ & & & & \\
\hline 4 & 4,5 & 12,55 & 57 & 256,5 & $3,06 \mathrm{E}+04$ & & & & \\
\hline 5 & 3,2 & 16,4 & 57 & 182,4 & $3,06 \mathrm{E}+04$ & & & & \\
\hline 6 & 1 & 18,5 & 57 & 53,86 & $3,06 \mathrm{E}+04$ & 18,5 & 3,14 & $2, \mathrm{E}+05$ \\
\hline 7 & 3 & 20,5 & 57 & 171 & $3,06 \mathrm{E}+04$ & & & & \\
\hline 8 & 4,5 & 24,25 & 31 & 139,5 & $3,06 \mathrm{E}+04$ & & & & \\
\hline 9 & 4,5 & 28,75 & 31 & 139,5 & $3,06 \mathrm{E}+04$ & & & & \\
\hline 10 & 4,5 & 33,25 & 31 & 139,5 & $3,06 \mathrm{E}+04$ & & & & \\
\hline 11 & 4,9 & 37,95 & 31 & 151,9 & $3,06 \mathrm{E}+04$ & & & & \\
\hline 12 & 1,6 & 41,2 & 31 & 45,58 & $3,06 \mathrm{E}+04$ & 41,2 & 4,02 & $2, \mathrm{E}+05$ \\
\hline 13 & 3 & 43,5 & 31 & 93 & $3,06 \mathrm{E}+04$ & & & & \\
\hline
\end{tabular}

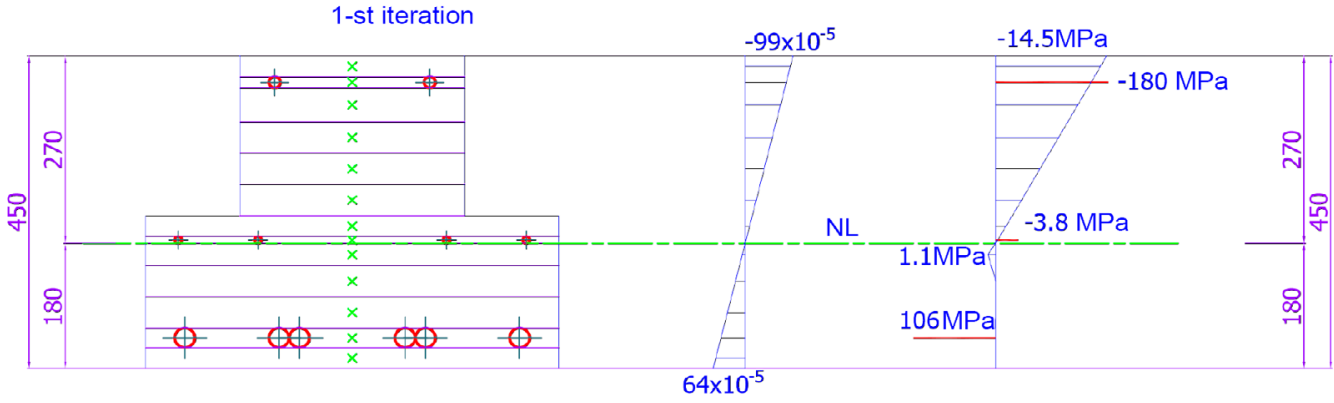

Figure 7. First iteration of the first method 
Table 2. First iteration of the first method

\begin{tabular}{|c|c|c|c|c|c|c|c|c|c|c|c|c|}
\hline \multirow{2}{*}{$i$} & $A \_b$ & $E \_b$ & $A_{-} S$ & $E \_s$ & $y_{-} o j$ & $y_{-} b$ & $y_{-} s$ & \multirow{2}{*}{$1 / \mathrm{cm}^{-1}$} & \multirow{2}{*}{$\varepsilon_{-} b$} & $\sigma_{-} b$ & \multirow{2}{*}{$\varepsilon_{-} S$} & $\sigma \_s$ \\
\hline & $\mathrm{cm}^{2}$ & $\mathrm{MPa}$ & $\mathrm{cm}^{2}$ & $\mathrm{MPa}$ & $\mathrm{cm}$ & $\mathrm{cm}$ & $\mathrm{cm}$ & & & $\mathrm{MPa}$ & & $\mathrm{MPa}$ \\
\hline 1 & 171 & $3.06 \mathrm{E}+04$ & & & \multirow{13}{*}{$\begin{array}{l}-1 \\
0 \\
\infty \\
\infty \\
\rightarrow-1\end{array}$} & 16.51 & & \multirow{13}{*}{$\begin{array}{l}\text { L } \\
\text { ' } \\
\dot{山} \\
\infty \\
\infty \\
\dot{m}\end{array}$} & $6.41 \mathrm{E}-04$ & 0.00 & & \\
\hline 2 & 122.8 & $3.06 E+04$ & 36.8 & 2.E+05 & & 13.61 & 13.61 & & $5.29 \mathrm{E}-04$ & 0.00 & $5.29 \mathrm{E}-04$ & 105.7 \\
\hline 3 & 256.5 & $3.06 E+04$ & & & & 9.96 & & & 3.87E-04 & 0.00 & & \\
\hline 4 & 256.5 & $3.06 E+04$ & & & & 5.46 & & & 2.12E-04 & 0.00 & & \\
\hline 5 & 182.4 & $3.06 E+04$ & & & & 1.61 & & & $6.26 \mathrm{E}-05$ & 1.10 & & \\
\hline 6 & 53.86 & $3.06 \mathrm{E}+04$ & 3.14 & 2. $E+05$ & & -0.49 & -0.49 & & $-1.89 E-05$ & -0.28 & $-1.89 E-05$ & -3.7846 \\
\hline 7 & 171 & $3.06 \mathrm{E}+04$ & & & & -2.49 & & & $-9.66 \mathrm{E}-05$ & -1.42 & & \\
\hline 8 & 139.5 & $3.06 \mathrm{E}+04$ & & & & -6.24 & & & $-2.42 \mathrm{E}-04$ & -3.55 & & \\
\hline 9 & 139.5 & $3.06 E+04$ & & & & -10.74 & & & $-4.17 \mathrm{E}-04$ & -6.11 & & \\
\hline 10 & 139.5 & $3.06 E+04$ & & & & -15.24 & & & $-5.92 E-04$ & -8.68 & & \\
\hline 11 & 151.9 & $3.06 \mathrm{E}+04$ & & & & -19.94 & & & $-7.74 \mathrm{E}-04$ & -11.35 & & \\
\hline 12 & 45.58 & $3.06 \mathrm{E}+04$ & 4.02 & 2.E+05 & & -23.19 & -23.19 & & $-9.00 E-04$ & -13.20 & $-9.00 \mathrm{E}-04$ & -180.05 \\
\hline 13 & 93 & $3.06 E+04$ & & & & -25.49 & & & $-9.90 E-04$ & -14.51 & & \\
\hline
\end{tabular}

Table 3. Second iteration of the first method

\begin{tabular}{|c|c|c|c|c|c|c|c|c|c|c|c|c|}
\hline \multirow{2}{*}{$i$} & $A \_b$ & $E \_b$ & $A \_s$ & $E_{-} S$ & $y_{-} o_{j}$ & $y_{-} b$ & $y_{-} s$ & \multirow{2}{*}{$1 / \mathrm{cm}^{-1}$} & \multirow{2}{*}{$\varepsilon_{-} b$} & $\sigma_{-} b$ & \multirow{2}{*}{$\varepsilon_{-} S$} & $\sigma_{-} S$ \\
\hline & $\mathrm{cm}^{2}$ & $\mathrm{MPa}$ & $\mathrm{cm}^{2}$ & $\mathrm{MPa}$ & $\mathrm{cm}$ & $\mathrm{cm}$ & $\mathrm{cm}$ & & & $\mathrm{MPa}$ & & $\mathrm{MPa}$ \\
\hline 1 & 171 & $0.00 \mathrm{E}+00$ & & & \multirow{13}{*}{$\begin{array}{l}0 \\
\stackrel{-}{\sim} \\
\stackrel{\sim}{N}\end{array}$} & 19.66 & & \multirow{13}{*}{ 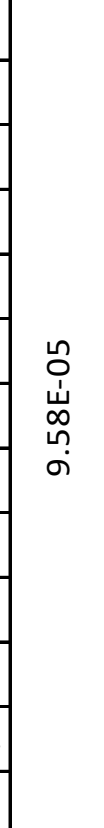 } & $1.88 \mathrm{E}-03$ & 0.00 & & \\
\hline 2 & 122.8 & $0.00 \mathrm{E}+00$ & 36.8 & 2.E+05 & & 16.76 & 16.76 & & $1.61 \mathrm{E}-03$ & 0.00 & $1.61 \mathrm{E}-03$ & 321.2 \\
\hline 3 & 256.5 & $0.00 \mathrm{E}+00$ & & & & 13.11 & & & $1.26 \mathrm{E}-03$ & 0.00 & & \\
\hline 4 & 256.5 & $0.00 \mathrm{E}+00$ & & & & 8.61 & & & $8.25 \mathrm{E}-04$ & 0.00 & & \\
\hline 5 & 182.4 & 1.75E+04 & & & & 4.76 & & & 4.56E-04 & 0.00 & & \\
\hline 6 & 53.86 & $1.47 \mathrm{E}+04$ & 3.14 & 2.E+05 & & 2.66 & 2.66 & & $2.55 \mathrm{E}-04$ & 0.00 & $2.55 \mathrm{E}-04$ & 50.977 \\
\hline 7 & 171 & $1.47 \mathrm{E}+04$ & & & & 0.66 & & & $6.32 \mathrm{E}-05$ & 1.11 & & \\
\hline 8 & 139.5 & $1.47 \mathrm{E}+04$ & & & & -3.09 & & & $-2.96 \mathrm{E}-04$ & -4.34 & & \\
\hline 9 & 139.5 & 1.47E+04 & & & & -7.59 & & & $-7.27 \mathrm{E}-04$ & -10.67 & & \\
\hline 10 & 139.5 & $1.47 \mathrm{E}+04$ & & & & -12.09 & & & $-1.16 \mathrm{E}-03$ & -16.99 & & \\
\hline 11 & 151.9 & $1.47 \mathrm{E}+04$ & & & & -16.79 & & & $-1.61 \mathrm{E}-03$ & -22.00 & & \\
\hline 12 & 45.58 & $1.47 \mathrm{E}+04$ & 4.02 & 2. $E+05$ & & -20.04 & -20.04 & & $-1.92 \mathrm{E}-03$ & -22.00 & $-1.92 \mathrm{E}-03$ & -355 \\
\hline 13 & 93 & $1.47 \mathrm{E}+04$ & & & & -22.34 & & & $-2.14 \mathrm{E}-03$ & -22.00 & & \\
\hline
\end{tabular}

of the lower stretched zone to the center of gravity, area and modulus of elasticity of $i$-n reinforcement, accordingly; $y_{0 j}=y_{01}=180 \mathrm{~mm}-$ position of neutral line in the course of the first iteration.

Tables 2-9 and Figure 5-12 show the results of iteration calculations according to the first and the second methods. 5:
$M_{\text {calc }}=\sum \sigma_{b i} A_{b i} y_{b i}^{\prime}+\sum \sigma_{s i} A_{s i} y_{s i}^{\prime}=424.459 \cdot 10^{6} \mathrm{~N} \mathrm{~mm}$

Height of compressed zone:

$x=h-y_{011}=45-21.09=24.91 \mathrm{~cm}$

Example of calculation according to the second iteration method 


\section{Architecture and Engineering Volume 3 Issue 3}

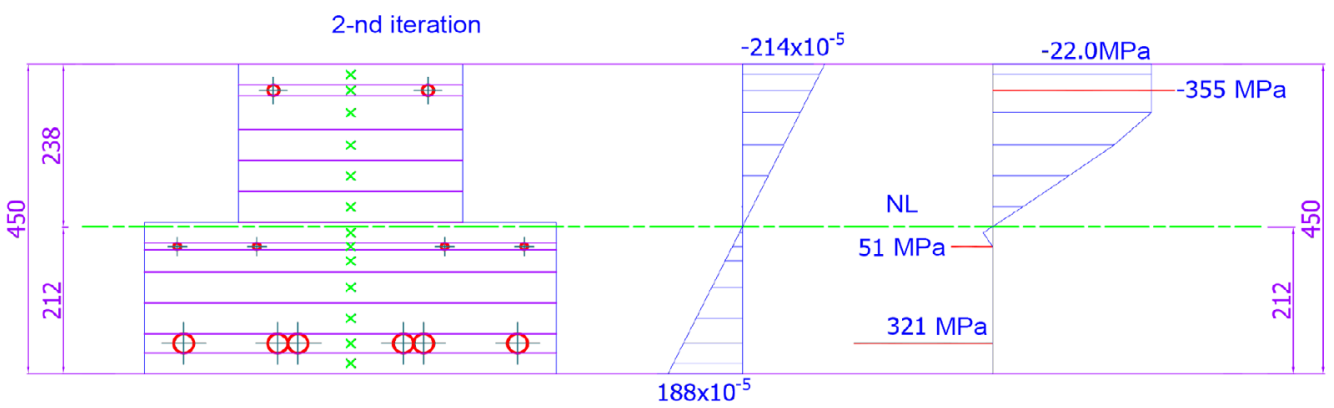

Figure 8. Second iteration of the first method

Table 4. Third iteration of the first method

\begin{tabular}{|c|c|c|c|c|c|c|c|c|c|c|c|c|}
\hline \multirow{2}{*}{$i$} & $A_{-} b$ & $E \_b$ & $A \_S$ & $E \_s$ & $y_{-} o_{j}$ & $y_{-} b$ & $y_{-} s$ & \multirow{2}{*}{ 1/ $\mathrm{cm}^{-1}$} & \multirow{2}{*}{$\varepsilon_{-} b$} & $\sigma_{-} b$ & \multirow{2}{*}{$\varepsilon_{-} S$} & $\sigma_{-} S$ \\
\hline & $\mathrm{cm}^{2}$ & $\mathrm{MPa}$ & $\mathrm{cm}^{2}$ & $\mathrm{MPa}$ & $\mathrm{cm}$ & $\mathrm{cm}$ & $\mathrm{cm}$ & & & $\mathrm{MPa}$ & & $\mathrm{MPa}$ \\
\hline 1 & 171 & $0.00 \mathrm{E}+00$ & & & \multirow{13}{*}{$\begin{array}{l}\stackrel{-}{\sim} \\
\stackrel{-}{v}\end{array}$} & 19.71 & & \multirow{13}{*}{ 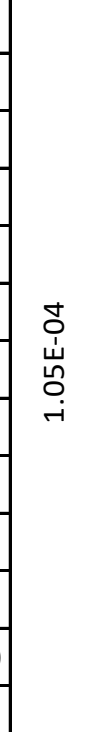 } & $2.08 \mathrm{E}-03$ & 0.00 & & \\
\hline 2 & 122.8 & $0.00 \mathrm{E}+00$ & 36.8 & 2. $E+05$ & & 16.81 & 16.81 & & 1.77E-03 & 0.00 & 1.77E-03 & 354.41 \\
\hline 3 & 256.5 & $0.00 E+00$ & & & & 13.16 & & & $1.39 \mathrm{E}-03$ & 0.00 & & \\
\hline 4 & 256.5 & $0.00 E+00$ & & & & 8.66 & & & 9.13E-04 & 0.00 & & \\
\hline 5 & 182.4 & $0.00 \mathrm{E}+00$ & & & & 4.81 & & & 5.07E-04 & 0.00 & & \\
\hline 6 & 53.86 & $0.00 E+00$ & 3.14 & 2. $E+05$ & & 2.71 & 2.71 & & $2.86 \mathrm{E}-04$ & 0.00 & 2.86E-04 & 57.219 \\
\hline 7 & 171 & $1.75 \mathrm{E}+04$ & & & & 0.71 & & & 7.53E-05 & 1.32 & & \\
\hline 8 & 139.5 & $1.47 \mathrm{E}+04$ & & & & -3.04 & & & $-3.20 \mathrm{E}-04$ & -4.69 & & \\
\hline 9 & 139.5 & $1.47 \mathrm{E}+04$ & & & & -7.54 & & & $-7.94 \mathrm{E}-04$ & -11.65 & & \\
\hline 10 & 139.5 & $1.47 \mathrm{E}+04$ & & & & -12.04 & & & $-1.27 \mathrm{E}-03$ & -18.60 & & \\
\hline 11 & 151.9 & $1.37 \mathrm{E}+04$ & & & & -16.74 & & & $-1.76 \mathrm{E}-03$ & -22.00 & & \\
\hline 12 & 45.58 & $1.15 \mathrm{E}+04$ & 4.02 & 2. $E+05$ & & -19.99 & -19.99 & & $-2.11 \mathrm{E}-03$ & -22.00 & $-2.11 \mathrm{E}-03$ & -355 \\
\hline 13 & 93 & $1.03 E+04$ & & & & -22.29 & & & $-2.35 E-03$ & -22.00 & & \\
\hline
\end{tabular}

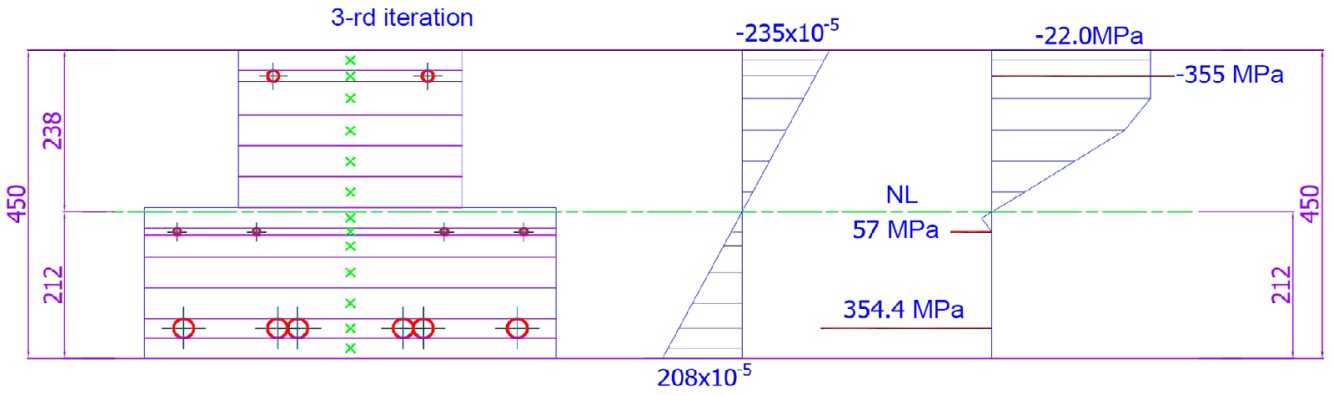

Figure 9. Third iteration of the first method

11-th iteration

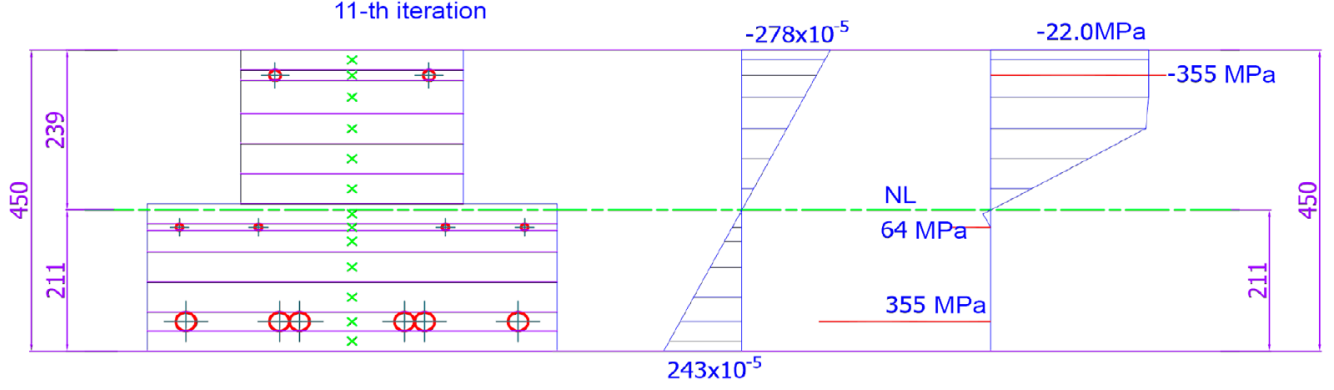

Figure 10. Eleventh iteration of the first method 
Table 5. Eleventh iteration of the first method

\begin{tabular}{|c|c|c|c|c|c|c|c|c|c|c|c|c|}
\hline \multirow{2}{*}{$i$} & $A_{-} b$ & $E_{-} b$ & $A_{-} S$ & $E \_S$ & $y_{-} o_{j}$ & $y_{-} b$ & $y_{-} s$ & \multirow{2}{*}{ 1/ $\mathrm{cm}^{-1}$} & \multirow{2}{*}{$\varepsilon_{-} b$} & $\sigma \_b$ & \multirow{2}{*}{$\varepsilon_{-} S$} & $\sigma_{-} S$ \\
\hline & $\mathrm{cm}^{2}$ & $\mathrm{MPa}$ & $\mathrm{cm}^{2}$ & $\mathrm{MPa}$ & $\mathrm{cm}$ & $\mathrm{cm}$ & $\mathrm{cm}$ & & & $\mathrm{MPa}$ & & MPa \\
\hline 1 & 171 & $0.00 \mathrm{E}+00$ & & & \multirow{13}{*}{$\begin{array}{l}\text { \& } \\
\text { ते }\end{array}$} & 19.59 & & \multirow{13}{*}{ 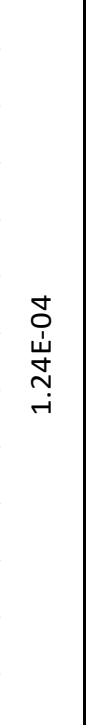 } & $2.43 \mathrm{E}-03$ & 0.00 & & \\
\hline 2 & 122.8 & $0.00 \mathrm{E}+00$ & 36.8 & 2. $E+05$ & & 16.69 & 16.69 & & 2.07E-03 & 0.00 & 2.07E-03 & 355 \\
\hline 3 & 256.5 & $0.00 \mathrm{E}+00$ & & & & 13.04 & & & $1.61 \mathrm{E}-03$ & 0.00 & & \\
\hline 4 & 256.5 & $0.00 \mathrm{E}+00$ & & & & 8.54 & & & $1.06 \mathrm{E}-03$ & 0.00 & & \\
\hline 5 & 182.4 & $0.00 \mathrm{E}+00$ & & & & 4.69 & & & $5.81 \mathrm{E}-04$ & 0.00 & & \\
\hline 6 & 53.86 & $0.00 \mathrm{E}+00$ & 3.14 & 2. $E+05$ & & 2.59 & 2.59 & & $3.21 \mathrm{E}-04$ & 0.00 & $3.21 \mathrm{E}-04$ & 64.175 \\
\hline 7 & 171 & $1.75 E+04$ & & & & 0.59 & & & 7.32E-05 & 1.28 & & \\
\hline 8 & 139.5 & $1.47 E+04$ & & & & -3.16 & & & $-3.91 E-04$ & -5.74 & & \\
\hline 9 & 139.5 & $1.47 \mathrm{E}+04$ & & & & -7.66 & & & $-9.48 E-04$ & -13.91 & & \\
\hline 10 & 139.5 & $1.47 \mathrm{E}+04$ & & & & -12.16 & & & $-1.51 E-03$ & -22.00 & & \\
\hline 11 & 151.9 & $1.06 \mathrm{E}+04$ & & & & -16.86 & & & $-2.09 E-03$ & -22.00 & & \\
\hline 12 & 45.58 & $8.92 \mathrm{E}+03$ & 4.02 & 1. E+05 & & -20.11 & -20.11 & & $-2.49 \mathrm{E}-03$ & -22.00 & $-2.49 \mathrm{E}-03$ & -355 \\
\hline 13 & 93 & $8.01 E+03$ & & & & -22.41 & & & $-2.78 \mathrm{E}-03$ & -22.00 & & \\
\hline
\end{tabular}

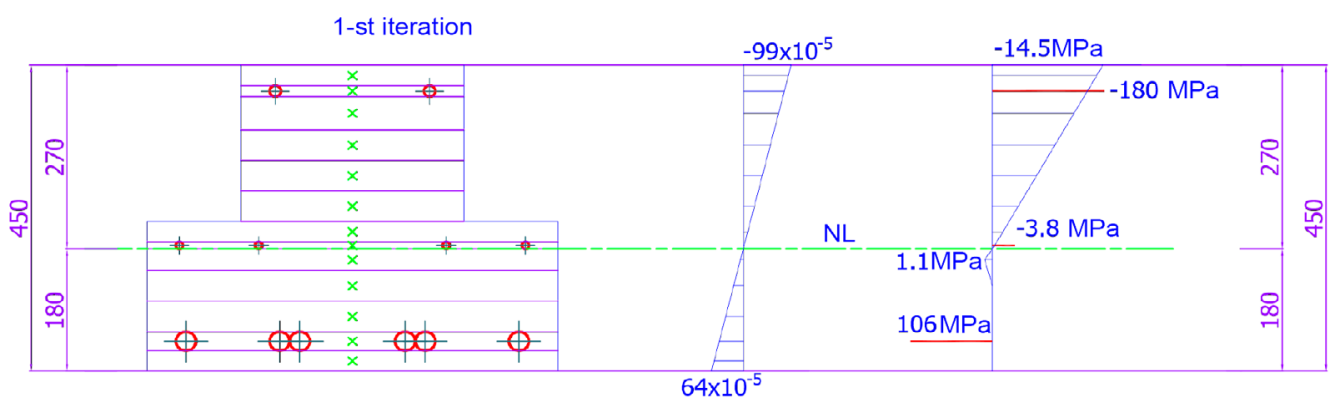

Figure 11. First iteration of the second method

Table 6. First iteration of the second method

\begin{tabular}{|c|c|c|c|c|c|c|c|c|c|c|c|c|}
\hline \multirow{2}{*}{$i$} & $\Delta$ & $y_{-} b$ & $b \_b$ & $A_{-} b$ & $E \_b$ & $y_{-} s$ & $A \_s$ & $E \_s$ & \multirow{2}{*}{$\varepsilon_{-} b$} & $\sigma_{-} b$ & \multirow{2}{*}{$\varepsilon_{-} S$} & $\sigma_{-} S$ \\
\hline & $\mathrm{cm}$ & $\mathrm{cm}$ & $\mathrm{cm}$ & $\mathrm{cm}^{2}$ & $\mathrm{MPa}$ & $\mathrm{cm}$ & $\mathrm{cm}^{2}$ & MPa & & $\mathrm{MPa}$ & & $\mathrm{MPa}$ \\
\hline 1 & 3 & 1.5 & 57 & 171 & $3.06 E+04$ & & & & $6.41 \mathrm{E}-04$ & 0.00 & & \\
\hline 2 & 2.8 & 4.4 & 57 & 122.8 & $3.06 E+04$ & 4.4 & 36.8 & $2 . E+05$ & $5.29 \mathrm{E}-04$ & 0.00 & $5.29 \mathrm{E}-04$ & 105.70 \\
\hline 3 & 4.5 & 8.05 & 57 & 256.5 & $3.06 E+04$ & & & & $3.87 \mathrm{E}-04$ & 0.00 & & \\
\hline 4 & 4.5 & 12.6 & 57 & 256.5 & $3.06 \mathrm{E}+04$ & & & & $2.12 \mathrm{E}-04$ & 0.00 & & \\
\hline 5 & 3.2 & 16.4 & 57 & 182.4 & $3.06 \mathrm{E}+04$ & & & & $6.26 \mathrm{E}-05$ & 1.10 & & \\
\hline 6 & 1 & 18.5 & 57 & 53.86 & $3.06 E+04$ & 18.5 & 3.14 & 2.E+05 & $-1.89 \mathrm{E}-05$ & -0.28 & $-1.89 \mathrm{E}-05$ & -3.78 \\
\hline 7 & 3 & 20.5 & 57 & 171 & $3.06 E+04$ & & & & $-9.66 \mathrm{E}-05$ & -1.42 & & \\
\hline 8 & 4.5 & 24.3 & 31 & 139.5 & $3.06 \mathrm{E}+04$ & & & & $-2.42 \mathrm{E}-04$ & -3.55 & & \\
\hline 9 & 4.5 & 28.8 & 31 & 139.5 & $3.06 \mathrm{E}+04$ & & & & $-4.17 \mathrm{E}-04$ & -6.11 & & \\
\hline 10 & 4.5 & 33.3 & 31 & 139.5 & $3.06 E+04$ & & & & $-5.92 \mathrm{E}-04$ & -8.68 & & \\
\hline 11 & 4.9 & 38 & 31 & 151.9 & $3.06 E+04$ & & & & $-7.74 \mathrm{E}-04$ & -11.35 & & \\
\hline 12 & 1.6 & 41.2 & 31 & 45.58 & $3.06 \mathrm{E}+04$ & 41.2 & 4.02 & 2.E+05 & $-9.00 \mathrm{E}-04$ & -13.20 & $-9.00 \mathrm{E}-04$ & -180.05 \\
\hline 13 & 3 & 43.5 & 31 & 93 & $3.06 \mathrm{E}+04$ & & & & $-9.90 \mathrm{E}-04$ & -14.51 & & \\
\hline
\end{tabular}


Architecture and Engineering Volume 3 Issue 3

Table 7. Second iteration of the second method

\begin{tabular}{|c|c|c|c|c|c|c|c|c|c|c|c|}
\hline \multirow{2}{*}{$i$} & $y_{-} b$ & $b \_b$ & $A_{-} b$ & $E \_b$ & $y_{-} s$ & $A \_S$ & $E \_s$ & \multirow{2}{*}{$\varepsilon_{-} b$} & $\sigma_{-} b$ & \multirow{2}{*}{$\varepsilon_{-} S$} & $\sigma_{-} S$ \\
\hline & $\mathrm{cm}$ & $\mathrm{cm}$ & $\mathrm{cm}^{2}$ & $\mathrm{MPa}$ & $\mathrm{cm}$ & $\mathrm{cm}^{2}$ & $\mathrm{MPa}$ & & $\mathrm{MPa}$ & & $\mathrm{MPa}$ \\
\hline 1 & 1.5 & 57 & 171 & $0.00 \mathrm{E}+00$ & & & & $1.88 \mathrm{E}-03$ & 0.00 & & \\
\hline 2 & 4.4 & 57 & 122.8 & $0.00 E+00$ & 4.4 & 36.8 & 2. $E+05$ & $1.61 \mathrm{E}-03$ & 0.00 & 1.61E-03 & 321.20 \\
\hline 3 & 8.05 & 57 & 256.5 & $0.00 \mathrm{E}+00$ & & & & $1.26 \mathrm{E}-03$ & 0.00 & & \\
\hline 4 & 12.6 & 57 & 256.5 & $0.00 \mathrm{E}+00$ & & & & $8.25 \mathrm{E}-04$ & 0.00 & & \\
\hline 5 & 16.4 & 57 & 182.4 & $1.75 \mathrm{E}+04$ & & & & 4.56E-04 & 0.00 & & \\
\hline 6 & 18.5 & 57 & 53.86 & 1.47E+04 & 18.5 & 3.14 & 2. $E+05$ & 2.55E-04 & 0.00 & 2.55E-04 & 50.98 \\
\hline 7 & 20.5 & 57 & 171 & 1.47E+04 & & & & $6.32 \mathrm{E}-05$ & 1.11 & & \\
\hline 8 & 24.3 & 31 & 139.5 & 1.47E+04 & & & & $-2.96 \mathrm{E}-04$ & -4.34 & & \\
\hline 9 & 28.8 & 31 & 139.5 & $1.47 \mathrm{E}+04$ & & & & $-7.27 E-04$ & $|-10.67|$ & & \\
\hline 10 & 33.3 & 31 & 139.5 & $1.47 \mathrm{E}+04$ & & & & $-1.16 \mathrm{E}-03$ & -16.99 & & \\
\hline 11 & 38 & 31 & 151.9 & 1.47E+04 & & & & $-1.61 \mathrm{E}-03$ & -22.00 & & \\
\hline 12 & 41.2 & 31 & 45.58 & 1.47E+04 & 41.2 & 4.02 & 2. $E+05$ & $-1.92 \mathrm{E}-03$ & -22.00 & $-1.92 \mathrm{E}-03$ & -355.00 \\
\hline 13 & 43.5 & 31 & 93 & 1.47E+04 & & & & $-2.14 \mathrm{E}-03$ & -22.00 & & \\
\hline
\end{tabular}

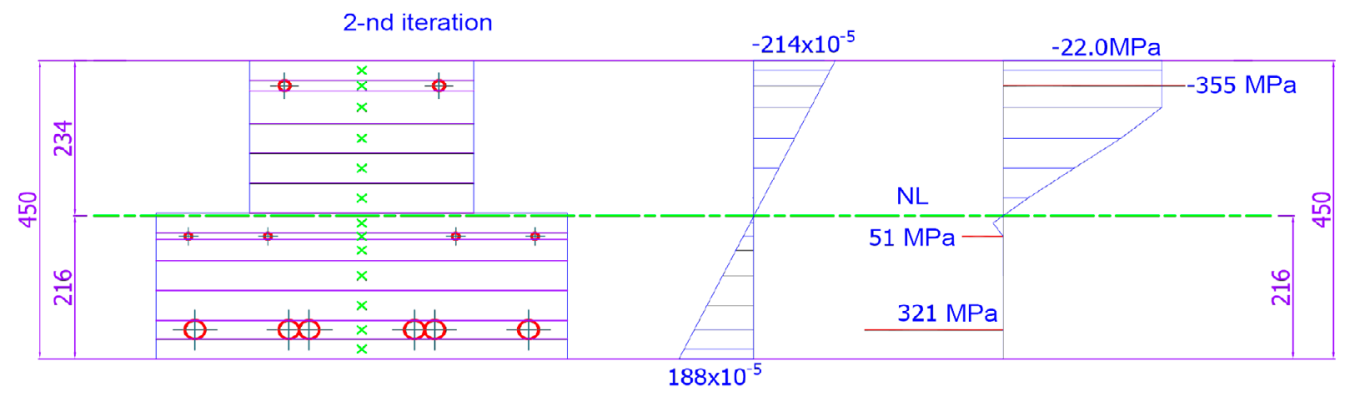

Figure 12. Second iteration of the second method

Table 8. Third iteration of the second method

\begin{tabular}{|c|c|c|c|c|c|c|c|c|c|c|c|}
\hline \multirow{2}{*}{$i$} & $y_{-} b$ & $b \_b$ & $A \_b$ & $E \_b$ & $y_{-} s$ & $A \_s$ & $E \_s$ & \multirow{2}{*}{$\varepsilon_{-} b$} & $\sigma_{-} b$ & \multirow{2}{*}{$\varepsilon_{-} S$} & $\sigma_{-} s$ \\
\hline & $\mathrm{cm}$ & $\mathrm{cm}$ & $\mathrm{cm}^{2}$ & $\mathrm{MPa}$ & $\mathrm{cm}$ & $\mathrm{cm}^{2}$ & $\mathrm{MPa}$ & & $\mathrm{MPa}$ & & $\mathrm{MPa}$ \\
\hline 1 & 1.5 & 57 & 171 & $0.00 E+00$ & & & & $2.08 \mathrm{E}-03$ & 0.00 & & \\
\hline 2 & 4.4 & 57 & 122.8 & $0.00 E+00$ & 4.4 & 36.8 & 2. $E+05$ & 1.77E-03 & 0.00 & 1.77E-03 & 354.41 \\
\hline 3 & 8.05 & 57 & 256.5 & $0.00 E+00$ & & & & 1.39E-03 & 0.00 & & \\
\hline 4 & 12.6 & 57 & 256.5 & $0.00 E+00$ & & & & $9.13 \mathrm{E}-04$ & 0.00 & & \\
\hline 5 & 16.4 & 57 & 182.4 & $0.00 E+00$ & & & & 5.07E-04 & 0.00 & & \\
\hline 6 & 18.5 & 57 & 53.86 & $0.00 E+00$ & 18.5 & 3.14 & 2. $E+05$ & $2.86 \mathrm{E}-04$ & 0.00 & $2.86 \mathrm{E}-04$ & 57.22 \\
\hline 7 & 20.5 & 57 & 171 & $1.75 \mathrm{E}+04$ & & & & 7.53E-05 & 1.32 & & \\
\hline 8 & 24.3 & 31 & 139.5 & $1.47 \mathrm{E}+04$ & & & & $-3.20 E-04$ & -4.69 & & \\
\hline 9 & 28.8 & 31 & 139.5 & $1.47 \mathrm{E}+04$ & & & & $-7.94 \mathrm{E}-04$ & -11.65 & & \\
\hline 10 & 33.3 & 31 & 139.5 & $1.47 \mathrm{E}+04$ & & & & $-1.27 \mathrm{E}-03$ & -18.60 & & \\
\hline 11 & 38 & 31 & 151.9 & $1.37 E+04$ & & & & $-1.76 \mathrm{E}-03$ & -22.00 & & \\
\hline 12 & 41.2 & 31 & 45.58 & $1.15 E+04$ & 41.2 & 4.02 & 2. $E+05$ & $-2.11 \mathrm{E}-03$ & -22.00 & $-2.11 \mathrm{E}-03$ & -355.00 \\
\hline 13 & 43.5 & 31 & 93 & $1.03 E+04$ & & & & $-2.35 \mathrm{E}-03$ & -22.00 & & \\
\hline
\end{tabular}




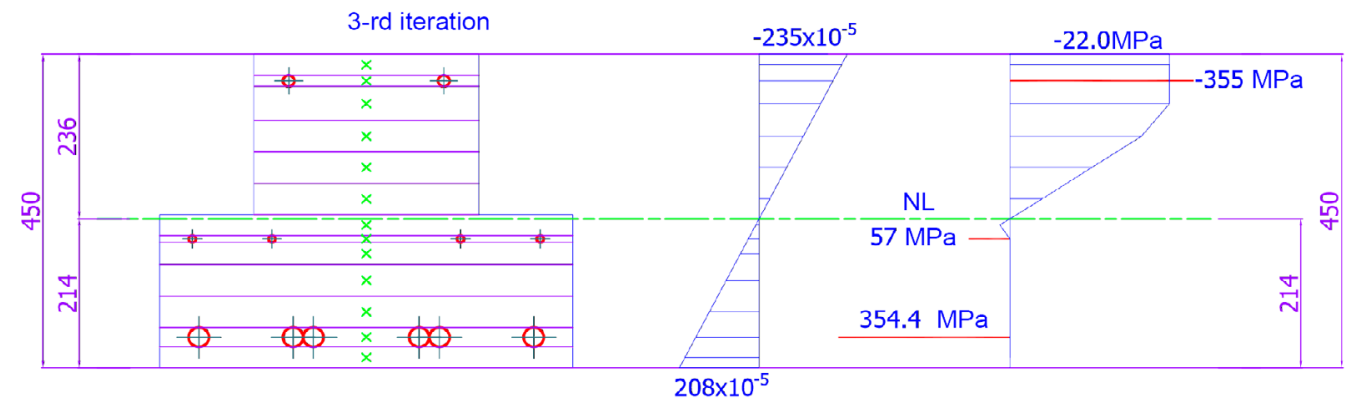

Figure 13. Third iteration of the second method

Table 9. Eleventh iteration of the second method

\begin{tabular}{|c|c|c|c|c|c|c|c|c|c|c|c|}
\hline \multirow[b]{2}{*}{$i$} & $y_{-} b$ & $b \_b$ & $A_{-} b$ & $E \_b$ & $y_{-} s$ & $A \_s$ & $E_{-} S$ & \multirow{2}{*}{$\varepsilon_{-} b$} & $\sigma_{-} b$ & \multirow{2}{*}{$\varepsilon_{-} S$} & $\sigma_{-} s$ \\
\hline & $\mathrm{cm}$ & $\mathrm{cm}$ & $\mathrm{cm}^{2}$ & $\mathrm{MPa}$ & $\mathrm{cm}$ & $\mathrm{cm}^{2}$ & $\mathrm{MPa}$ & & $\mathrm{MPa}$ & & $\mathrm{MPa}$ \\
\hline 1 & 1.5 & 57 & 171 & $0.00 \mathrm{E}+00$ & & & & $2.40 \mathrm{E}-03$ & 0.00 & & \\
\hline 2 & 4.4 & 57 & 122.8 & $0.00 \mathrm{E}+00$ & 4.4 & 36.8 & $2 . E+05$ & 2.04E-03 & 0.00 & 2.04E-03 & 355.00 \\
\hline 3 & 8.05 & 57 & 256.5 & $0.00 \mathrm{E}+00$ & & & & 1.59E-03 & 0.00 & & \\
\hline 4 & 12.6 & 57 & 256.5 & $0.00 \mathrm{E}+00$ & & & & 1.04E-03 & 0.00 & & \\
\hline 5 & 16.4 & 57 & 182.4 & $0.00 E+00$ & & & & $5.70 \mathrm{E}-04$ & 0.00 & & \\
\hline 6 & 18.5 & 57 & 53.86 & $0.00 \mathrm{E}+00$ & 18.5 & 3.14 & 2.E+05 & $3.12 \mathrm{E}-04$ & 0.00 & $3.12 \mathrm{E}-04$ & 62.40 \\
\hline 7 & 20.5 & 57 & 171 & $1.75 E+04$ & & & & $6.66 \mathrm{E}-05$ & 1.17 & & \\
\hline 8 & 24.3 & 31 & 139.5 & $1.47 \mathrm{E}+04$ & & & & $-3.94 \mathrm{E}-04$ & -5.77 & & \\
\hline 9 & 28.8 & 31 & 139.5 & $1.47 \mathrm{E}+04$ & & & & $-9.46 \mathrm{E}-04$ & -13.87 & & \\
\hline 10 & 33.3 & 31 & 139.5 & $1.47 \mathrm{E}+04$ & & & & $-1.50 \mathrm{E}-03$ & -21.97 & & \\
\hline 11 & 38 & 31 & 151.9 & $1.07 E+04$ & & & & $-2.07 \mathrm{E}-03$ & -22.00 & & \\
\hline 12 & 41.2 & 31 & 45.58 & $8.99 \mathrm{E}+03$ & 41.2 & 4.02 & 1.E+05 & $-2.47 \mathrm{E}-03$ & -22.00 & $-2.47 \mathrm{E}-03$ & -355.00 \\
\hline 13 & 43.5 & 31 & 93 & 8.07E+03 & & & & $-2.76 \mathrm{E}-03$ & -22.00 & & \\
\hline
\end{tabular}

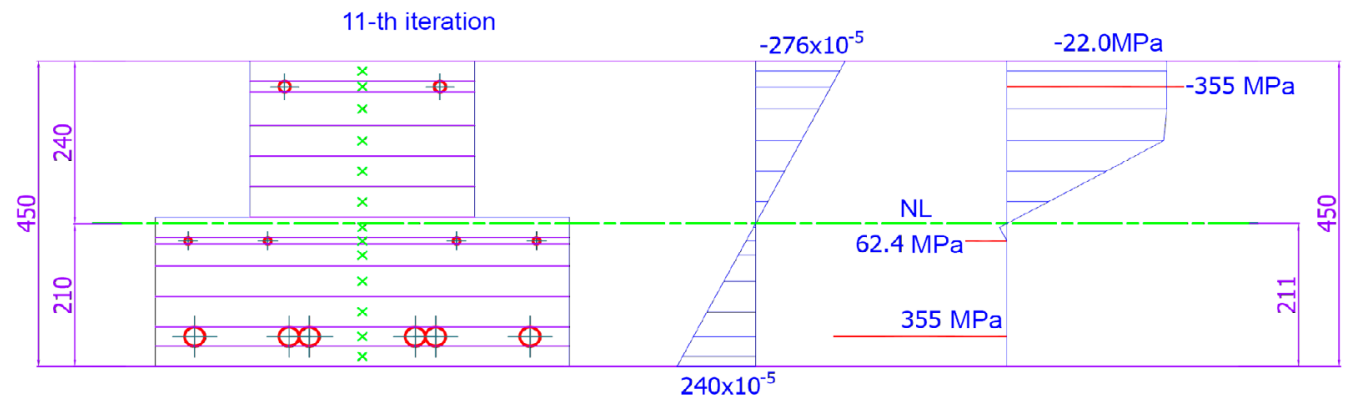

Figure 14. Eleventh iteration of the second method 
The relative deformations get determined depending on stiffness characteristics of every small area. The determination of height of compressed zone and graphical presentation of strain-stress state of cross section in the course of every iteration have been built depending on the calculated values of stresses and deformations with adherence to scale.

Table 10. Comparison of calculation parameters.

\begin{tabular}{|l|l|l|l|}
\hline $\begin{array}{l}\text { Calculation } \\
\text { method }\end{array}$ & Deformations & $\begin{array}{l}\text { Load-carry- } \\
\text { ing ability, } \\
\mathrm{kNm}\end{array}$ & $\begin{array}{l}\text { Height of } \\
\text { com- } \\
\text { pressed } \\
\text { zone, } \mathrm{cm}\end{array}$ \\
\hline $\begin{array}{l}\text { With respect to } \\
\text { limiting condi- } \\
\text { tions }\end{array}$ & - & 430.0 & 18.7 \\
\hline $\begin{array}{l}\text { Deformation } \\
\text { calculation 1 }\end{array}$ & $\begin{array}{l}\varepsilon_{b c, \text { alc }}=2.78 \cdot 10^{-3}<\left[\varepsilon_{b 2}=3.5 \cdot 10^{-3}\right] \\
\varepsilon_{s, \text { calc }}=0.00207<\left[\varepsilon_{s 2}=0.025\right]\end{array}$ & 424.5 & 24.91 \\
\hline $\begin{array}{l}\text { Deformation } \\
\text { calculation 2 }\end{array}$ & $\begin{array}{l}\varepsilon_{b c, \text { all }}=2.76 \cdot 10^{-3}<\left[\varepsilon_{b 2}=3.5 \cdot 10^{-3}\right] \\
\varepsilon_{s, \text { alc }}=0.00204<\left[\varepsilon_{s 2}=0.025\right]\end{array}$ & 427.8 & 24.06 \\
\hline
\end{tabular}

The height of compressed zone following a principle of "similarity of triangles" will be determined according to:

$x_{j}=\frac{h}{\frac{\varepsilon_{1}}{\left|\varepsilon_{13}\right|}+1}$

Thus, the summary limiting moment according to Table 9:

$M_{\text {calc }}=\sum \sigma_{b i} A_{b i} y_{b i}^{\prime}+\sum \sigma_{s i} A_{s i} y_{s i}^{\prime}=427.795 \cdot 10^{6} \mathrm{~N} \mathrm{~mm}$

We determine a height of the compressed zone through deformations using "indicators of similarity of triangles":

$x=\frac{h}{1.86956}=\frac{45}{1.86956}=24.06 \mathrm{~cm}$

\section{Conclusion}

The results presented in Table 10 testify to the fact that the acquired design values of deformations feature insignificant differences in case of calculations according to methods 1 and 2 and become adequately consistent with calculation data with respect to limiting conditions, which also proves adequacy of the developed methods. 


\section{References}

Boujelben, A., Ibrahimbegovic, A. (2017). Finite-strain three-dimensional solids with rotational degrees of freedom: non-linear statics and dynamics). Advanced Modeling and Simulation in Engineering Sciences, 4 (1), 3. DOI: 10.1186/s40323-017-0089-9

Dalla Valle, P., Thom, N. (2018). Improvement to method of equivalent thicknesses (MET) for calculation of critical strains for flexible pavements. International Journal of Pavement Engineering, 19 (12), pp. 1053-1060. DOI: 10.1080/10298436.2016.1238698

Fatehiboroujeni, S., Palanthandalam-Madapusi, H.J., Goyal, S. (2018). Computational Rod Model with User-Defined Nonlinear Constitutive Laws. Journal of Computational and Nonlinear Dynamics, 13( 10), 101006. DOI: 10.1115/1.4041028

Jagtap, K.R., Lal, A., Singh, B.N. (2018). Uncertainty quantification in non-linear dynamic response of functionally graded materials plate. Mechanics of Advanced Materials and Structures, 25 (13), pp. 1081-1100. DOI: 10.1080/15376494.2017.1329465

Kong, G., Zhou, Y., Liu, H. (2018). Nonlinear model analysis of radial bulging deformation of geosynthetic-encased stone columns. International Journal of Geomechanics, 18 (10), 06018022. DOI: 10.1061/(ASCE)GM.1943-5622.0001195

Mao, J.-J., Zhang, W. (2018). Linear and nonlinear free and forced vibrations of graphene reinforced piezoelectric composite plate under external voltage excitation. Composite Structures, 203, pp. 551-565. DOI: 10.1016/j.compstruct.2018.06.076

Minstroy RF (2013). SP 63.13330.2012. Concrete and reinforced-concrete structures. Basic provisions. Updated issue of SNiP 52-01-2003 (with revision Nos 1, 2), Moscow. (in Russian)

Morozov, V.I., Opbul, E.K. (2016). Raschet izgibaemyh stalefibrozhelezobetonnyh ehlementov po nelinejnoj deformacionnoj modeli s sipol'zovaniem opytnyh diagramm deformirovaniya stalefibrobetona [Calculation of bending steel fiber reinforced-concrete members according to non-linear deformation with the use of experimental diagrams of deformation of steel fiber reinforced-concrete]. Vestnik grazhdanskikh ingenerov [Bulletin of Civil Engineers], 5 (58), pp. 51-55. (in Russian)

Opbul, E.K., Dmitriev, D.A., Vedernikova, A.A. (2017). Nelinejno-iteracionnyj raschet prochnosti stalefibrozhelezobetonnyh ehlementov s sipol'zovaniem opytnyh diagramm deformirovaniya materialov [Non-linear and iteration calculation of strength of steel fiber reinforced-concrete members with the use of experimental diagrams of materials deformation]. Vestnik grazhdanskikh ingenerov [Bulletin of Civil Engineers], 1 (60), pp. 79-91. (in Russian)

Patni, M., Minera, S., Groh, R.M.J., Pirrera, A., Weaver, P.M. (2018). Three-dimensional stress analysis for laminated composite and sandwich structures. Composites Part B: Engineering, 155, pp. 299-328. DOI: 10.1016/j.compositesb.2018.08.127

TsRIIPromzdaniy OJSC (2005). Guidebook for designing concrete and reinforced-concrete structures from heavy concrete without pre-stressing (to SP 52-101-2003), Moscow. (in Russian) 